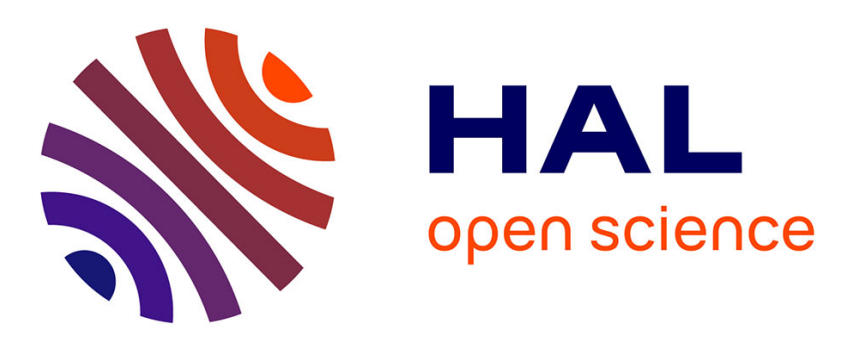

\title{
Delay induced high order locking effects in semiconductor lasers
}

B. Kelleher, M. Wishon, A. Locquet, D. Goulding, B. Tykalewicz, G. Huyet, E. Viktorov

\section{- To cite this version:}

B. Kelleher, M. Wishon, A. Locquet, D. Goulding, B. Tykalewicz, et al.. Delay induced high order locking effects in semiconductor lasers. Chaos: An Interdisciplinary Journal of Nonlinear Science, 2017, 27 (11), pp.114325. 10.1063/1.4994029 . hal-03014489

\section{HAL Id: hal-03014489 \\ https://cnrs.hal.science/hal-03014489}

Submitted on 19 Nov 2020

HAL is a multi-disciplinary open access archive for the deposit and dissemination of scientific research documents, whether they are published or not. The documents may come from teaching and research institutions in France or abroad, or from public or private research centers.
L'archive ouverte pluridisciplinaire HAL, est destinée au dépôt et à la diffusion de documents scientifiques de niveau recherche, publiés ou non, émanant des établissements d'enseignement et de recherche français ou étrangers, des laboratoires publics ou privés. 
Delay induced high order locking effects in semiconductor lasers

B. Kelleher, M. J. Wishon, A. Locquet, D. Goulding, B. Tykalewicz, G. Huyet, and E. A. Viktorov

Citation: Chaos 27, 114325 (2017);

View online: https://doi.org/10.1063/1.4994029

View Table of Contents: http://aip.scitation.org/toc/cha/27/11

Published by the American Institute of Physics 


\title{
Delay induced high order locking effects in semiconductor lasers
}

\author{
B. Kelleher, ${ }^{1,2}$ M. J. Wishon, ${ }^{3,4}$ A. Locquet, ${ }^{3,4}$ D. Goulding, ${ }^{2,5}$ B. Tykalewicz, ${ }^{2,5}$ G. Huyet, ${ }^{6,7}$ \\ and E. A. Viktorov 7,8 \\ ${ }^{1}$ Department of Physics, University College Cork, Cork, Ireland \\ ${ }^{2}$ Tyndall National Institute, University College Cork, Lee Maltings, Dyke Parade, Cork, Ireland \\ ${ }^{3}$ Georgia Tech-CNRS UMI 2958, Georgia Tech Lorraine, 2 Rue Marconi, 57070 Metz, France \\ ${ }^{4}$ School of Electrical and Computer Engineering, Georgia Institute of Technology, Atlanta, \\ Georgia 30332-0250, USA \\ ${ }^{5}$ Centre for Advanced Photonics and Process Analysis, Cork Institute of Technology, Cork, Ireland \\ ${ }^{6}$ Université Côte d'Azur, CNRS, Institut de Physique de Nice, Valbonne F-06560, France \\ ${ }^{7}$ National Research University of Information Technologies, Mechanics and Optics, \\ 49 Kronverksky Pr., St Petersburg 197101, Russia \\ ${ }^{8}$ Optique Nonlinéaire Théorique, Université libre de Bruxelles, Campus de la Plaine, CP 231, 1050 Bruxelles, \\ Belgium
}

(Received 3 July 2017; accepted 23 October 2017; published online 9 November 2017)

\begin{abstract}
Multiple time scales appear in many nonlinear dynamical systems. Semiconductor lasers, in particular, provide a fertile testing ground for multiple time scale dynamics. For solitary semiconductor lasers, the two fundamental time scales are the cavity repetition rate and the relaxation oscillation frequency which is a characteristic of the field-matter interaction in the cavity. Typically, these two time scales are of very different orders, and mutual resonances do not occur. Optical feedback endows the system with a third time scale: the external cavity repetition rate. This is typically much longer than the device cavity repetition rate and suggests the possibility of resonances with the relaxation oscillations. We show that for lasers with highly damped relaxation oscillations, such resonances can be obtained and lead to spontaneous mode-locking. Two different laser types-_a quantum dot based device and a quantum well based device - are analysed experimentally yielding qualitatively identical dynamics. A rate equation model is also employed showing an excellent agreement with the experimental results. Published by AIP Publishing. https://doi.org/10.1063/1.4994029
\end{abstract}

The semiconductor laser undergoing feedback is one of the most well-studied, non-linear laser configurations. It is of vital importance for applications given the ubiquity of lasers in modern technology and it is also the source of many fundamental non-linear phenomena. Semiconductor lasers have two intrinsic timescales: the cavity repetition rate (set primarily by the length of the laser) and the relaxation oscillation frequency (describing the rate at which energy is transferred between the electric field and the charge carriers). These two timescales are very different in typical devices. The relaxation oscillation frequency is typically on the order of a few $\mathrm{GHz}$ while the cavity repetition rate is often many tens of GHz. While nonlinear systems involving multiple time scales often lead to resonant effects, the vast separation in these two timescales means this is not typically observed with semiconductor lasers. By including delayed optical feedback, a third timescale is introducedthe external cavity repetition rate. This is often much lower than the solitary cavity repetition rate. We show here that for lasers with highly damped relaxation oscillations, resonances between the relaxation oscillations and the external cavity modes can and do occur and indeed, lead to spontaneous mode-locking. In fact, high order locking effects are possible with multipulse intensity trains.

\section{INTRODUCTION}

One of the most important and well-studied configurations for semiconductor lasers is that of external optical feedback. ${ }^{1}$
In this configuration, light from a laser is returned to the cavity by an external reflector after some delay time, typically of ns order and thus relevant for semiconductor physics. The practical reasons for its study are clear: Semiconductor lasers are ubiquitous in modern technology and since it is extremely difficult to remove all extraneous reflections, the behaviour of such devices undergoing feedback must be examined. External optical feedback can act as a stabilising influence on laser emission, allowing for narrow linewidths for single section lasers $^{2}$ and reduced jitter in passively mode locked devices. ${ }^{3,4}$ However, despite this beneficial feature, feedback is generally undesirable with conventional semiconductor lasers as typically, it leads to instabilities. ${ }^{1}$ These arise even for very low levels of feedback and very often lead to chaotic operation. ${ }^{5-7}$ Several material and device parameters are responsible for this sensitivity. Most notably, the linewidth enhancement factor (the $\alpha$ factor) and the weakly damped relaxation oscillations (ROs). The $\alpha$ factor quantifies the phase-amplitude coupling in a semiconductor device, arising due to the dependence of the refractive index on the carrier density of a semiconductor material. More pertinent to this work is the latter RO based phenomenon. If either the intensity or the carriers are perturbed in a semiconductor laser, the return to the steady state operation is via damped harmonic oscillations known as the ROs. They represent the back and forth exchange of energy between the field and the carriers in the device and the frequency of these oscillations yields one of the most important characteristic timescales in any perturbative configuration (such as optical feedback). For conventional semiconductor lasers the damping 
is typically very low. This is often quantified by the ratio of the RO damping to the RO frequency itself (just as in the case of the damped harmonic oscillator). For conventional devices, this ratio can be on the order of 0.01 or less. Thus, it is relatively easy to excite the ROs with an external forcing. This is central to instabilities both in feedback and optical injection configurations where excitation of the ROs is often the first step to chaos.

A second important timescale is given by the delay time $T$ : the time taken for the light to travel from the device to the external reflector and back to the device again. The external cavity induced by this configuration leads to the creation of external cavity modes (ECMs). For large delay time, these ECMs are separated by approximately $2 \pi / T$.

In conventional cases, the ROs of the device are excited by the feedback and become self-sustained. Further increases of the feedback strength then lead to coherence collapse (CC), a dramatic broadening of the laser linewidth distinguished by chaotic intensity pulsations. ${ }^{5,8} \mathrm{We}$ are not concerned with $\mathrm{CC}$ in this work but we simply note that there are several routes to $\mathrm{CC}$, including both period doubling and quasiperiodic ${ }^{9-11}$ and that in some cases this involves an interaction between ECMs and excited ROs and in other cases it might not. Further, the route to chaos is influenced by the initial conditions as shown recently in Ref. 12. In this work, we consider the possibility of mode-locking and optical comb generation via mutual resonances between the RO frequency (or harmonics) and ECMs.

With devices based on InAs QD material, the RO damping is significantly higher than with conventional devices and this leads to much more stable operation when undergoing optical feedback. ${ }^{13,14}$ The threshold feedback strength for the appearance of any instabilities rises dramatically and some phenomena including low frequency fluctuations $(\mathrm{LFF})^{7,15}$ do not arise at all. Recently, it has been observed that quantum cascade $(\mathrm{QC})$ lasers also display a very high stability in feedback configurations and in fact may be overdamped and potentially even Class A lasers that do not display relaxation oscillations at all. ${ }^{16-18}$

In Ref. 19, a resonance between the ROs and the ECMs was observed using a long external cavity and an InAs based QD device. It led to a resonant mode-locking where the RO frequency and a harmonic of the round-trip frequency coincided yielding a periodic train of pulses. As the pump current is increased, the RO frequency increases and thus, by examining the system at different pump currents one should obtain different resonance conditions. This evolution was investigated and, indeed, ratios from 1:5 to 1:11 were obtained. In this work, we go much further. We examine the evolution of the pulse train found in Ref. 19 as the feedback strength is increased, finding a splintering of modal groups leading eventually to chaos. To investigate the generality of the phenomenon, we also examine a different device type-a multiquantum well device with a high RO damping. Again, we find the generation of mode-locked traces via resonances between the ROs and ECMs. We reproduce our findings using a rate equation model and explain the observations physically.

\section{QUANTUM DOT DEVICE}

\section{A. Experiment}

For the first configuration, we use a quantum dot based device as in Ref. 19 with a threshold of $67 \mathrm{~mA}$. It operates from a single longitudinal mode with a side mode suppression ratio of greater than $40 \mathrm{~dB}$. The linewidth of the device was on the order of $1 \mathrm{MHz}$ as measured using a heterodyne technique. The external cavity is $48 \mathrm{~cm}$ long so that the external cavity repetition rate (ECRR) - the ECM separation-is approximately $313 \mathrm{MHz}$. The feedback strength $\varepsilon$ is adjusted using a variable neutral density optical filter. The output is analysed using a realtime digital oscilloscope and a fast photodetector of $12 \mathrm{GHz}$ bandwidth. A schematic of the setup is shown in Fig. 1.

The laser was biased at $79 \mathrm{~mA}$ and the feedback strength varied. As the filter is made progressively more transparent eventually, an instability is observed at a feedback strength of approximately $-16 \mathrm{~dB}$. This manifests as a small amplitude, almost harmonic, intensity time series which we interpret as arising via a Hopf bifurcation. Figures 2(a) and 2(b) show the intensity and the associated RF spectrum. The RF spectrum is dominated by peaks at the RO frequency and harmonics thereof. By measuring the frequency of this series as the pump current is increased, one can thus, in principle, find the Hopf frequency. Naively, one might expect this to map out a simple square root plot following the RO frequency dependence on the current. Indeed, in a preliminary examination of this frequency, this is indeed what was found. ${ }^{19}$ However, by measuring more closely and with a finer current step size, the situation is actually more detailed, as shown in Fig. 3.

Rather than in a smooth square root fashion, the frequency evolves in a series of discrete steps. Along each step, the frequency is almost constant and the frequency difference between consecutive steps corresponds to the round trip frequency. By fitting to the average frequency of each step, an overlaying square root dependence is found. The interpretation of the result is clear. The unlocking (Hopf) frequency is primarily governed by the RO frequency just as in the standard QW system. However, there is a mutual frequency pulling between the ROs and the underlying ECMs and this forces the frequency to almost coincide with the closest ECM frequency yielding a stepwise evolution. The

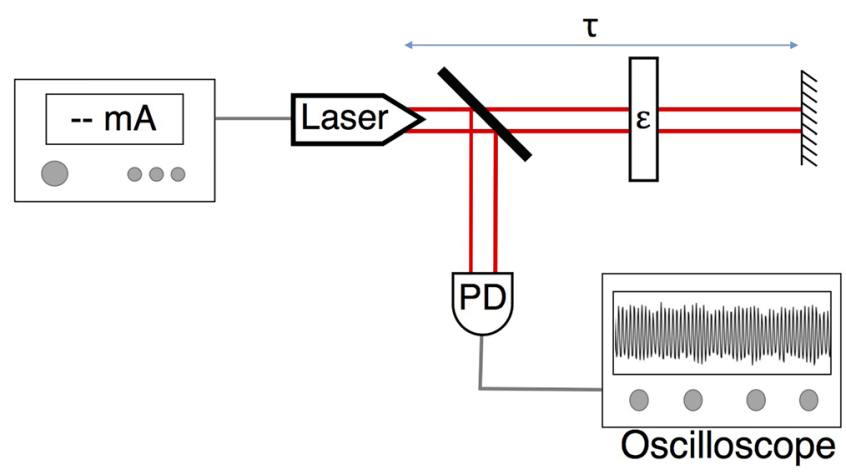

FIG. 1. Schematic of the experimental setup. OSA is an optical spectrum analyser. The feedback strength $\varepsilon$ is controlled by the transmission of the variable neutral density filter (labelled as $\varepsilon$ ). PD is a photodetector. 


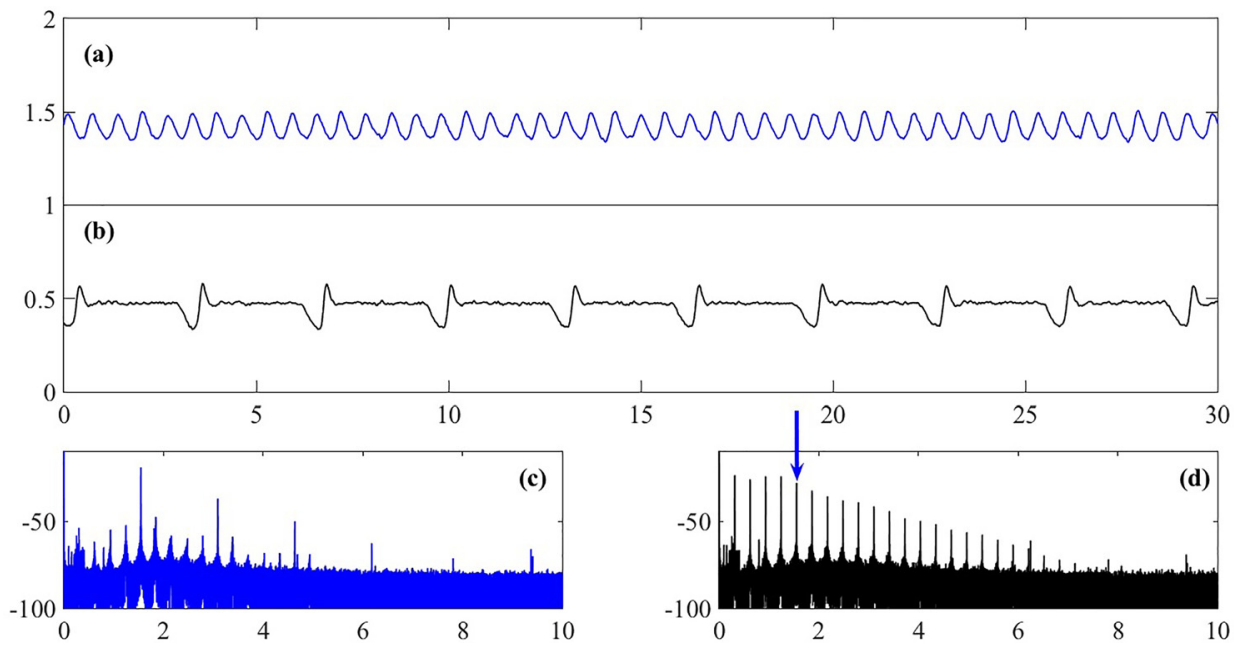

FIG. 2. (a) The low amplitude, almost harmonic intensity arising from the first instability. (b) A single pulse train with approximately round trip period. (c) The FFT spectrum corresponding to (a). (d) The FFT spectrum corresponding to (d). The arrow indicates the position of the RO frequency in this spectrum. frequency hardly changes along any one step before undergoing a discrete hop to the next step. It should be noted that experimentally it is extremely difficult to identify the exact point of the instability and so one cannot easily conclude on the exact Hopf frequency. Rather, one can only say that very close to the instability, the frequency of the time series is pinned and forced to lie on one of the observed steps.

\section{Time series evolution}

Returning to the $80 \mathrm{~mA}$ bias current and further increasing the feedback strength, the time series is modified. First, a train of single pulses separated by the round trip time is obtained as shown in Fig. 2(b). ${ }^{20}$ There is a distinctive shape to the pulses with a familiar trailing edge plateau as seen in several previous studies of quantum dot lasers. ${ }^{21,22}$ The RF spectrum now contains many peaks, separated by the round trip frequency.

As the feedback is further increased the intensity evolves to progressively more complicated multipulse trains as shown in Figs. 4 and 5. Moving from (a) through (c) in Fig. 4, one can see a double pulse train, a triple pulse train, and a quadruple pulse train. Figure 5 shows the corresponding RF spectra. In each case, the period of the train seems to be the round-trip delay. We interpret these trains as corresponding to winding rotations around a torus resulting from multiple Hopf bifurcations arising, as the feedback strength is increased. We note that by varying the operating current we have observed even higher orders of multipulse trains. The RF spectra changes in each case although not in any dramatic way. All the peaks in the RF spectra are narrow, indicating a high level of coherence.

Increasing the feedback further, the output is a train of oscillations with a frequency close to that of the ROs. For even higher levels of feedback, the system displays complex, likely chaotic, fluctuations as shown in Fig. 6(a). The associated RF spectrum in Fig. 6(b) is very broad, supporting the claim of chaos. Very small round trip peaks just higher than the broad pedestal are visible. There is clear evidence of multistability at high levels of feedback with different traces observed for different experimental runs. Multistability is also clearly visible at high currents even for moderate feedback levels.

As with the previous studies of such trailing edge plateau pulse trains in QD lasers, ${ }^{21,22}$ we interpret the shape as arising from modal groupings; in this case, groupings of ECMs. In this interpretation, one (or a few) main modes (close to the free-running mode) are associated with the plateau in each pulse while the other modes then contribute to the peak. As the feedback strength is further increased, the

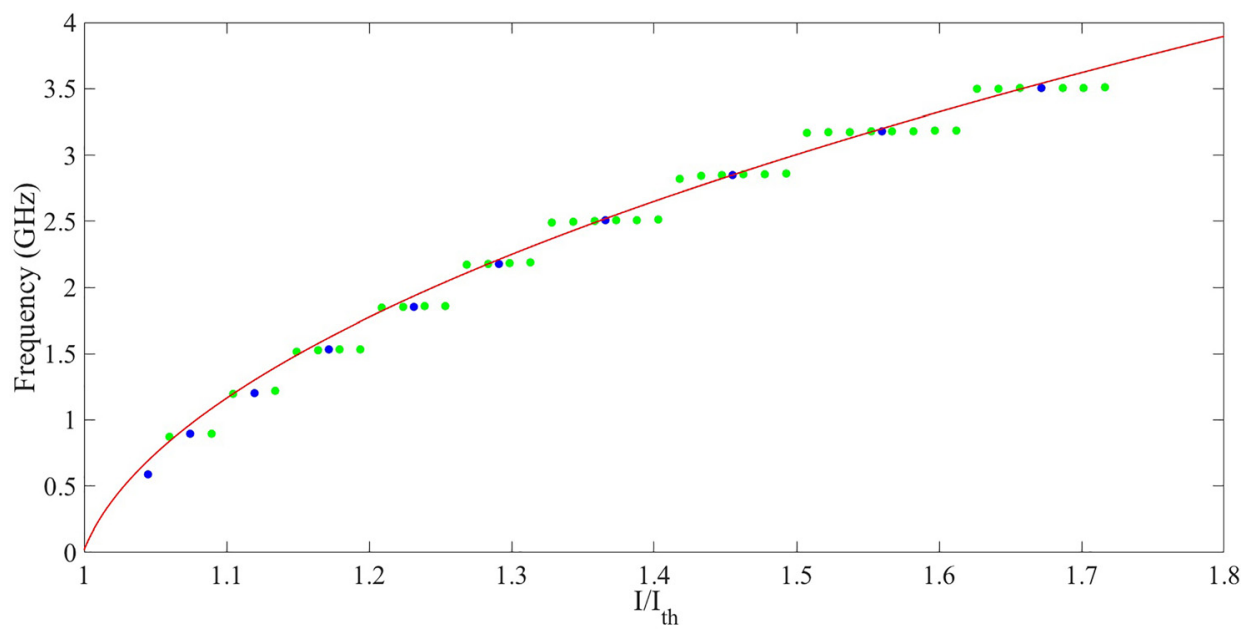

FIG. 3. The frequency of the first instability as a function of the pump current divided by the threshold current. There is a stepwise evolution with an overarching square root dependence. The circles label the measurements. The blue circles represent the "midpoints" of each step and these were the points used for the square root fit in red. 


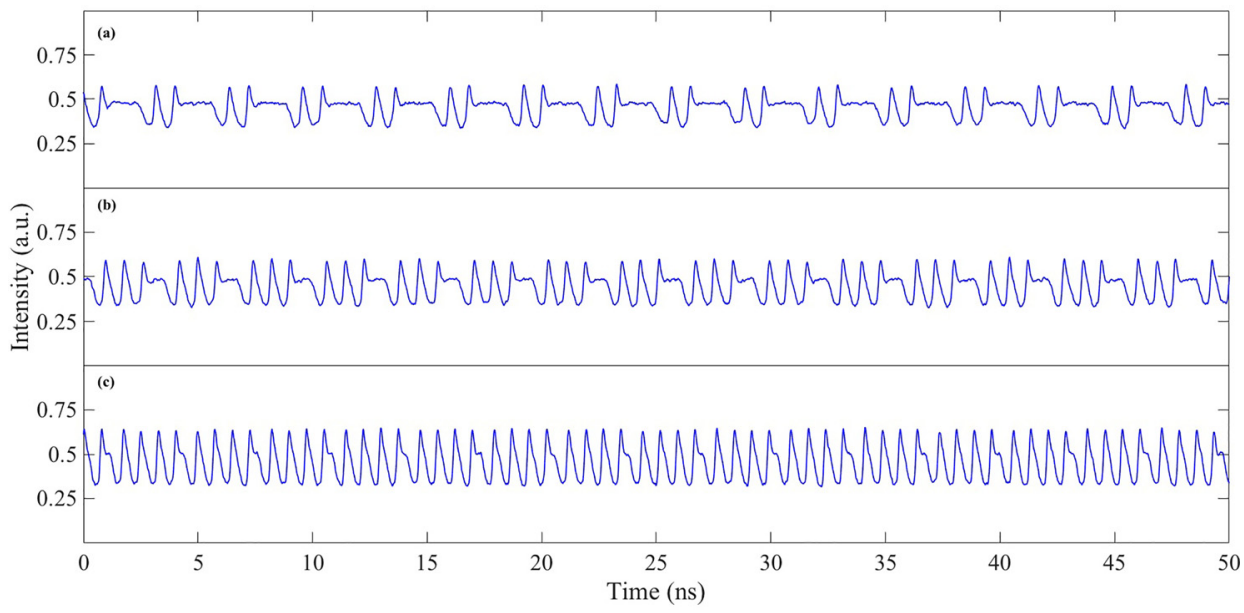

FIG. 4. (a) A round trip periodic double pulse train, (b) a round trip periodic triple pulse train, and (c) a round trip periodic quadruple pulse train.

groups splinter into smaller subgroups leading to a breakup of the plateau and multipulse trains. We verify this interpretation in the model via numerical frequency filtering.

\section{Coherence}

While the traces described and displayed thus far all appear to be round-trip periodic, it is instructive to analyse the coherence of the trains. We do this by analysing the linewidth of the main peaks in the RF spectra. The results are somewhat illuminating.

Figure 7 shows a zoom of the main peak in the single pulse RF spectrum and an associated best fit assuming a Lorentzian shape. The width of the fit is approximately $8.7 \mathrm{kHz}$. This suggests an excellent phase-locking of the ECMs. In fact, it is comparable with (and potentially lower than) the RF linewidth of the main peak obtained for freerunning, passively mode locked semiconductor lasers. ${ }^{3,23}$ Figure 8 shows a Lorentzian fit to the main peak in the RF spectrum for the double pulse train. A dramatic broadening is evident with a linewidth of almost $100 \mathrm{kHz}$ obtained in this case. By analysing the main peaks in the other multipulse trains a similar broadening is also obtained. (We also analysed the peak corresponding to the round trip frequency in each case and qualitatively identical results were obtained.) This suggests that the plateau breakup induces a decoherence in the pulse train, reminiscent of a previous result using a passively mode locked QD based laser. ${ }^{22}$ Regardless of interpretation, it is clear that the single pulse train is significantly more coherent than the subsequent multipulse trains.

In fact, further evidence of this can be obtained by direct inspection of the intensity trains. While extremely long traces can be found with one and only one multipulse type present, evolving trains are also observed. Figure 9 shows one such case where a triple pulse evolves into a double pulse via the disappearance of the leading peak. No such observations were found for the single pulse train. Thus, we conclude that the single pulse train is both more stable and more coherent than the more complex multipulse trains typically associated with higher feedback strength.

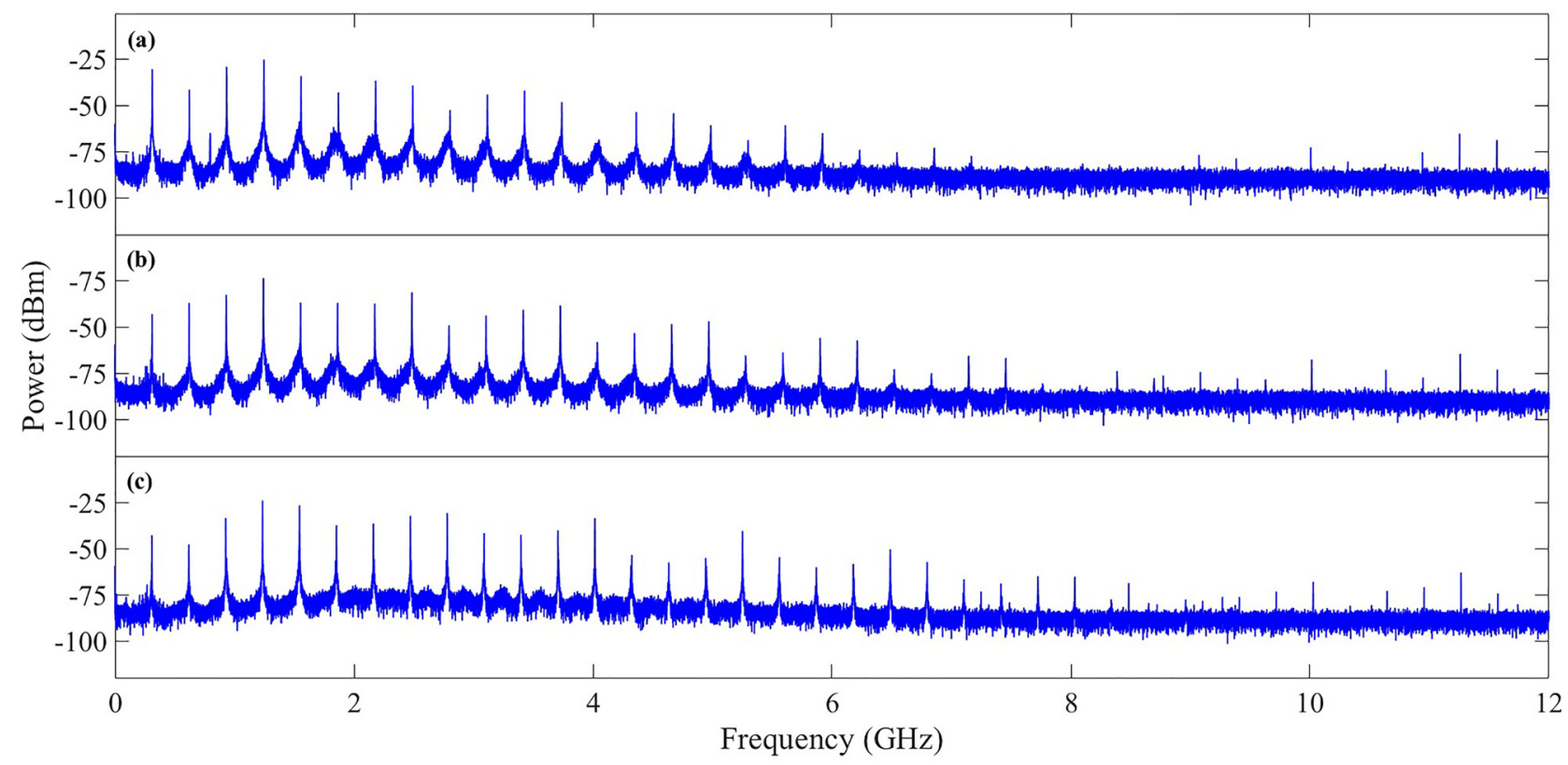

FIG. 5. (a)-(c) The respective FFT spectra corresponding to the intensity traces shown in Fig. 4. 

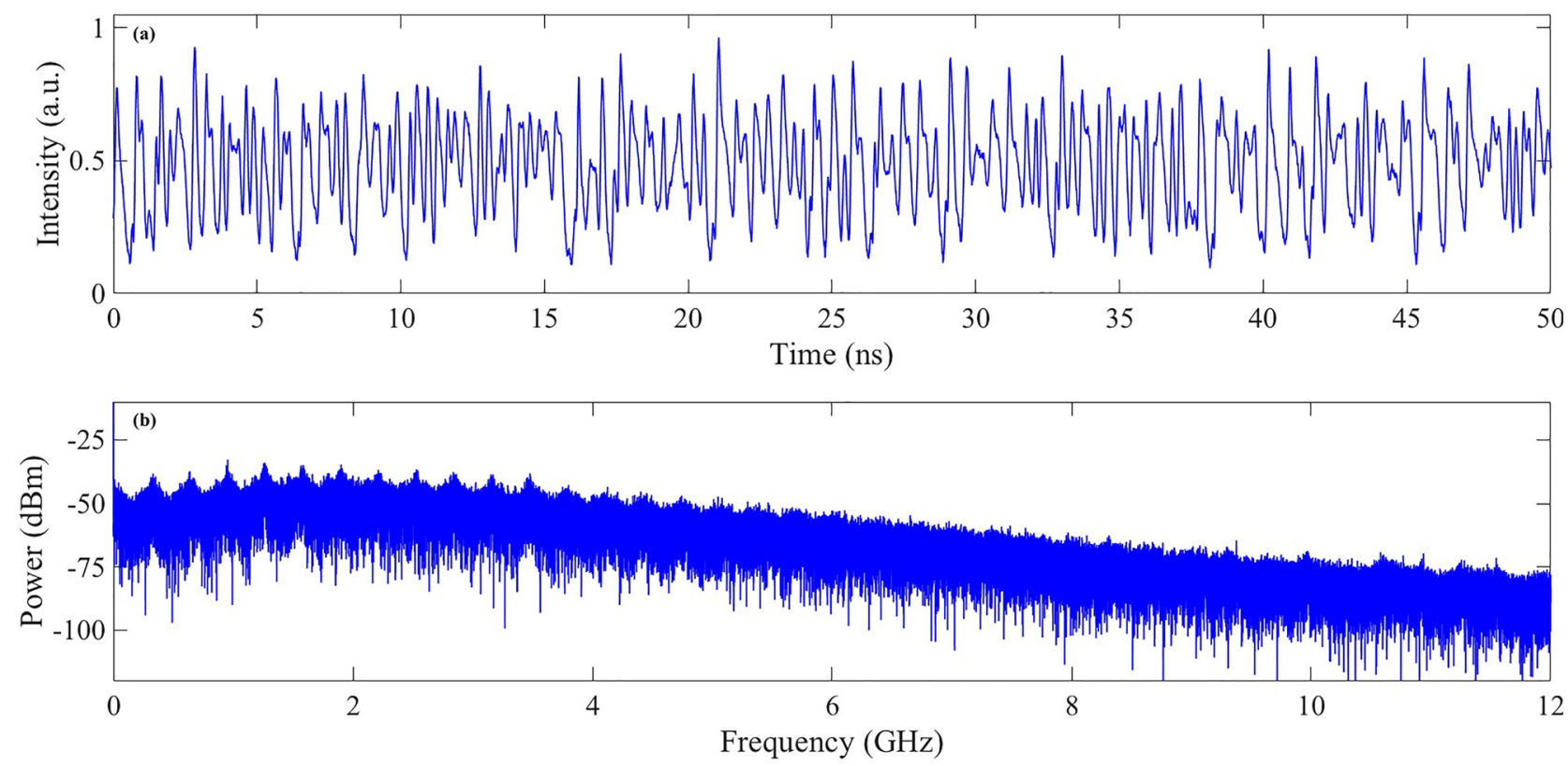

FIG. 6. (a) The intensity in a chaotic regime. (b) The corresponding FFT spectrum.

\section{B. Model}

We model the behaviour using a dimensionless delay differential equation system. The semiconductor laser itself of length $L_{\text {int }}$ is short and contains the gain medium. The external cavity, of length $L_{\text {ext }} \gg L_{\text {int }}$, is empty. The evolution of the normalized complex amplitude of the electric field $E(t)$ is given by

$$
\begin{aligned}
\gamma^{-1} \frac{d E}{d t}+E(t)= & \sqrt{\kappa} \exp ((1-i \alpha) G(t-\tau) / 2+i \varphi) \\
& \times E(t-\tau)+\varepsilon \exp ((1-i \alpha) G(t-\tau-T) / 2 \\
& +i \psi) E(t-\tau-T),
\end{aligned}
$$

as introduced in Ref. 19 as a generalization of the field equation in Refs. 4 and 24. Time $t$ is normalized by the short cavity round trip time. The dimensionless time delays $\tau$ and $T$

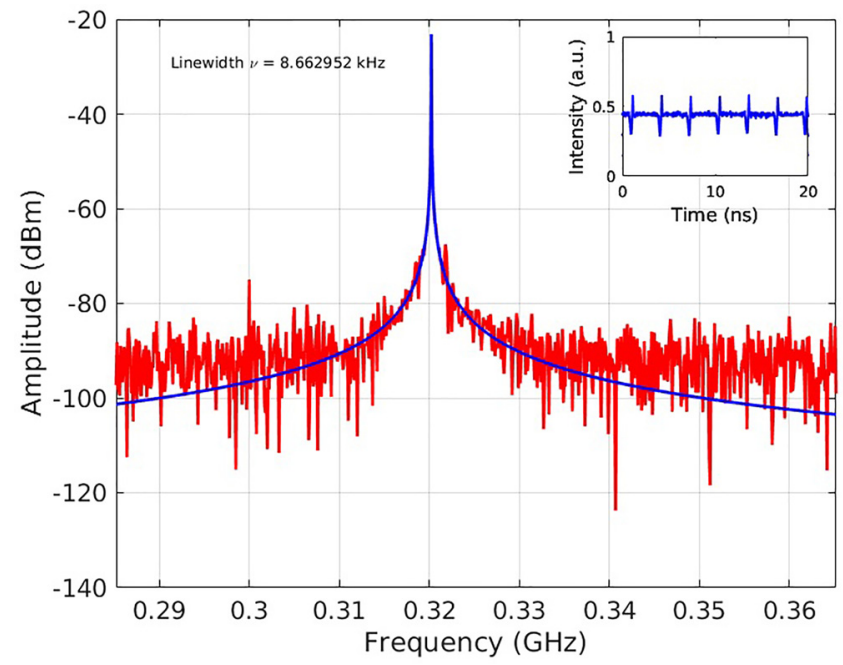

FIG. 7. Zoom of the main peak in the single pulse spectrum (red) with the best fit Lorentzian (blue) overlaid. The inset shows a small portion of the intensity time series. The legend shows the linewidth of the fit. correspond to the short and long cavity round trip times, determined by the cavity lengths $L_{\text {int }}$ and $L_{\text {ext }}$, respectively. The attenuation factor $\kappa<1$ describes total non-resonant linear intensity losses per cavity round trip, $\gamma$ is the dimensionless narrow bandwidth of the short laser cavity that matches the condition of single mode operation, and $\alpha$ is the linewidth enhancement factor. $\varepsilon$ quantifies the feedback strength. $\varphi$ is a control parameter that describes the difference between the frequency at which the gain is maximum and the optical frequency of the closest short cavity mode. $\psi$ is a control parameter that describes the difference between the frequency at which the gain is maximum and the optical frequency of the closest external cavity mode. $G(t)$ is the normalized gain defined by $G(t) \equiv 2 g L_{\text {int }}\left[2 \rho_{g}(t)-1\right]$, where $g$ is the effective gain factor. The evolution of the occupation probabilities satisfies the following equations:

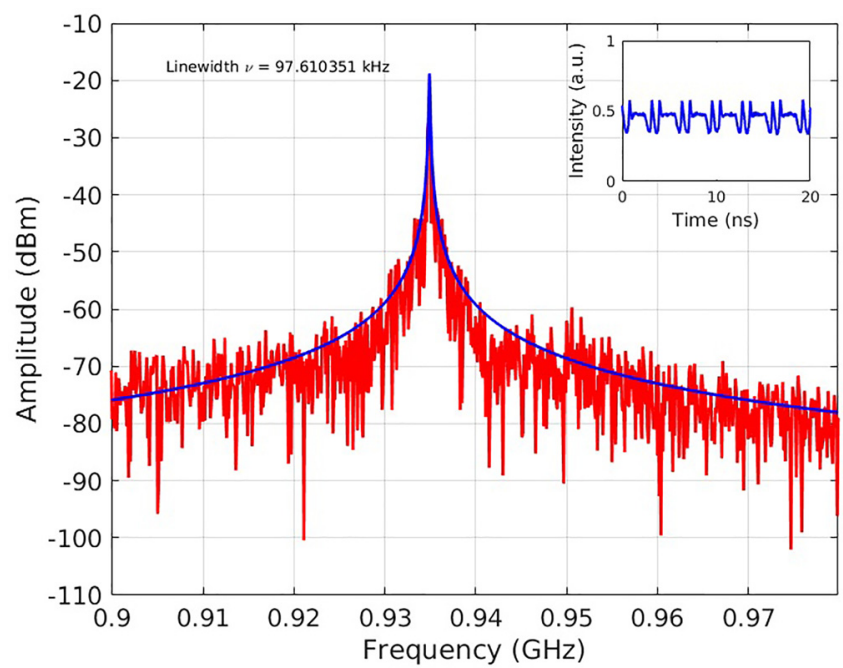

FIG. 8. Zoom of the main peak in the double pulse spectrum (red) with the best fit Lorentzian (blue) overlaid. The inset shows a small portion of the intensity time series. The legend shows the linewidth of the fit. 

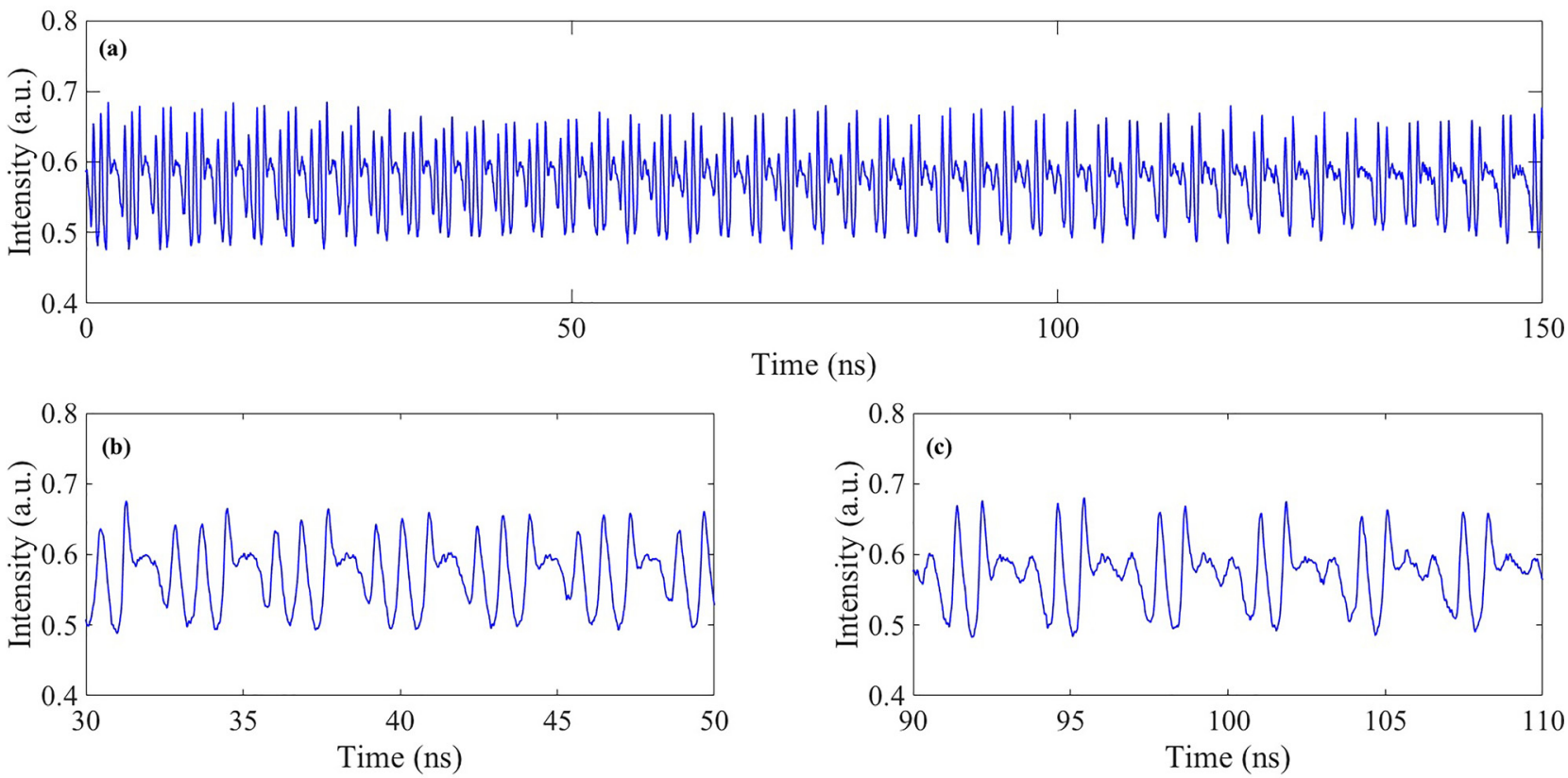

FIG. 9. An example of an evolving multipulse train. (a) A section where a triple pulse transforms into a double pulse, while (b) and (c) zooms of the two multipulse types.

$$
\begin{gathered}
\eta^{-1} \frac{d \rho_{g}}{d t}=-\rho_{g}+2 F\left(\rho_{g}, \rho_{e}\right)-\left(e^{G}-1\right)|E|^{2}, \\
\eta^{-1} \frac{d \rho_{e}}{d t}=-\rho_{e}-F\left(\rho_{g}, \rho_{e}\right)+D\left(\rho_{e}, N\right), \\
\eta^{-1} \frac{d N}{d t}=N_{0}-N-4 D\left(\rho_{e}, N\right),
\end{gathered}
$$

where $\rho_{g}(t)$ and $\rho_{e}(t)$ describe the ground $(\mathrm{GS})$ and excited (ES) state dot occupation probabilities and $N(t)$ describes the carrier density in the wetting layer, scaled to the QD carrier density. $\eta \equiv \tau \tau_{c}^{-1} \ll 1$ where $\tau_{c}$ denotes the carrier recombination time. The dimensionless parameter $N_{0}$ describes the pumping process in the gain section. The factors 2 and 4 in Eqs. (2) and (4) account for the spin degeneracy in the quantum dot energy levels. The functions $D\left(\rho_{e}, N\right)$ and $F\left(\rho_{g}, \rho_{e}\right)$ describe the carrier exchange rate between the wetting layer and the ES of the dots and the carrier exchange between the GS and ES of the dots:

$$
\begin{gathered}
D\left(\rho_{e}, N\right)=R_{w}^{c a p}\left(1-\rho_{e}\right)-R_{w}^{e s c} \rho_{e}, \\
F\left(\rho_{g}, \rho_{e}\right)=R^{c a p}\left(1-\rho_{g}\right)-R^{e s c}\left(1-\rho_{e}\right),
\end{gathered}
$$

where $R_{w}^{c a p} \equiv B N$ describes the carrier capture from the wetting layer to the dots with rate $B . R_{w}^{e s c}$ is a temperaturedependent coefficient that corresponds to the carrier escape from the dots to the wetting layer. $R^{c a p} \equiv B \rho_{e}$ and $R^{e s c} \equiv C \rho_{g}$ define the energy exchange between the GS and ES. The $\left(1-\rho_{e, g}\right)$ factors describe Pauli blocking in the expressions (5) and (6).

The numerical parameters were chosen in order to match the RO frequency and the external cavity roundtrip time of the QD laser configuration used in the experiment: $\eta=\tau \tau_{c}^{-1}$ $=0.01$, where $\tau=10 \mathrm{ps}$ is the short cavity roundtrip time and $\tau_{c} \sim 1000 \mathrm{ps}$ is the recombination time. For the other parameters, we use $\alpha=4, B=1000, C=100, \gamma=1.2$, $R_{w}^{e s c}=10,2 g L_{\mathrm{int}}=4, \tau=1$, and $\tau+T=21 \gg \tau$.

Direct simulations reproduce the experimental findings extremely well. For low feedback strengths, the output has a constant intensity. At the onset of the first instability, a Hopf bifurcation occurs, roughly corresponding physically to an undamping of the relaxation oscillations. This yields coherent sidebands around the central optical frequency. The frequency of the resulting trains is close to the RO frequency but is in resonance with external cavity mode frequencies explaining the stairlike dependence in Fig. 4. The amplitude of the oscillations in the sinusoidal trace at the RO frequency is small and remains small with increasing pump current. A second Hopf bifurcation then arises at the roundtrip frequency and the sidebands that result here can inject and excite neighbouring external cavity modes. It leads to quasiperiodic oscillations with the slow envelope frequency being the ECRR similar to that discussed experimentally.

The resonance between the RO frequency and the ECRR yields a trailing edge pulse (TEP) $\operatorname{train}^{21}$ in Fig. 10. As with the experiment, the ratio between the RO frequency and the external cavity frequency can be varied depending on the cavity length and pump current, but the correspondence with the experiment is excellent.

As the pump current is increased, more complex patterns were obtained. These include the evolution from single to multipulse trains similar to those observed in the experiment. Figure 10 shows a numerically calculated duplet and evolving traces of multipulse trains. There is a period doubling between the first trace and the second which is observable in the changing height of the second peak in each double pulse. We note that experimentally, such subtle changes may not be visible due to noise. Numerically, we find that the system is highly multistable with different pulse trains possible for the same control parameters. This issue is extremely complex and 


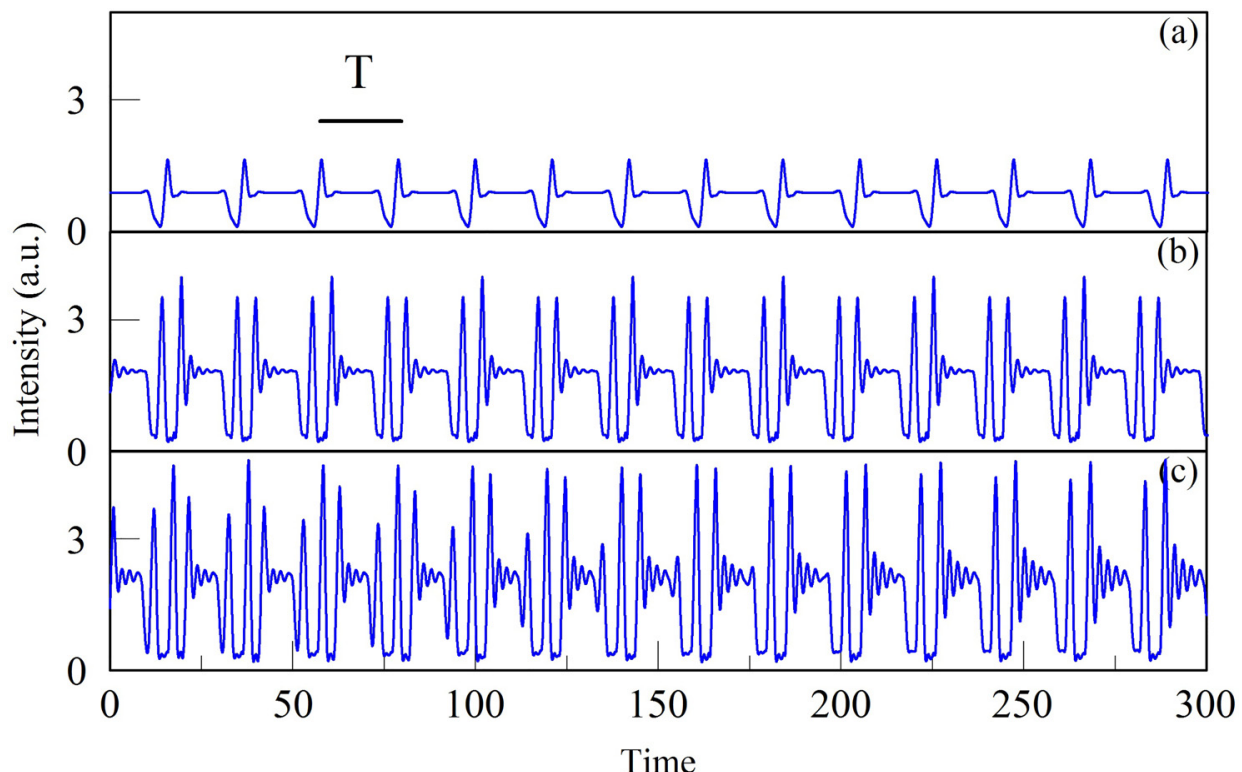

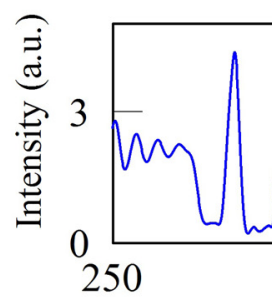

Time

we do not attempt to analyse it further in this work. We note that variations of the phases $\varphi$ and $\psi$ and $\varphi$ may affect the pulse shapes and the locking ranges. However, our aim in this work is to demonstrate that the experimentally observed phenomenon is also found in the theory, rather than an in-depth analysis of the locking ranges and the detailed effects of varying these phases.

For the higher order pulse trains, the stability and coherence degrade. This is visible in direct simulation of the intensity via the loss of periodicity and also, as shown in Fig. 10, where a triple pulse becomes a double pulse, in an excellent agreement with the experimental result of Fig. 9 .

The similarity of the fundamental train with the pulses obtained in Ref. 21 is very suggestive and motivates a modal analysis. We use a numerical filter to analyse the contribution to the output from different parts of the optical spectrum. For numerical expediency, we use a Lorentzian filter placed at different frequencies. The lower (red and blue) curves in Fig. 11 show the results of filtering different sections. When the filter is located at the highest power modes, the output matches the plateaux to a good approximation while there is almost no contribution to the peak. When the filter is located at one side of the spectrum, a more traditional mode-locked trace is obtained with short pulses followed by almost constant and closer to zero intensity. Thus, we conclude that the plateaux and peaks are formed by different groups of modes. Each group may have its own particular phase relationship between the modes, different in general to other groups. Nonetheless, the relative phases are fixed across the spectrum. This mode grouping phenomenon appears to be somewhat generic for

FIG. 10. Numerical traces. (a) A single pulse train with approximately round trip period. (b) A periodic double pulse train with approximately doubled round trip period. The period doubling can be seen by examining the height of the second peak in each double pulse. (c) An evolving multipulse train where triple pulse (d) transforms into a double pulse (e). The parameters are $N_{0}=100$ (a); $N_{0}=200 \quad$ (b); $N_{0}=250 \quad$ (c); $\kappa=0.3125, \varepsilon=0.25$, and $\varphi=\psi=0$. recently again been observed with QD passively mode-locked lasers in Ref. 22 where it leads to the formation of a chimera state. In this work, it represents a spontaneous mode-locking, resulting in a periodic pulse-train.

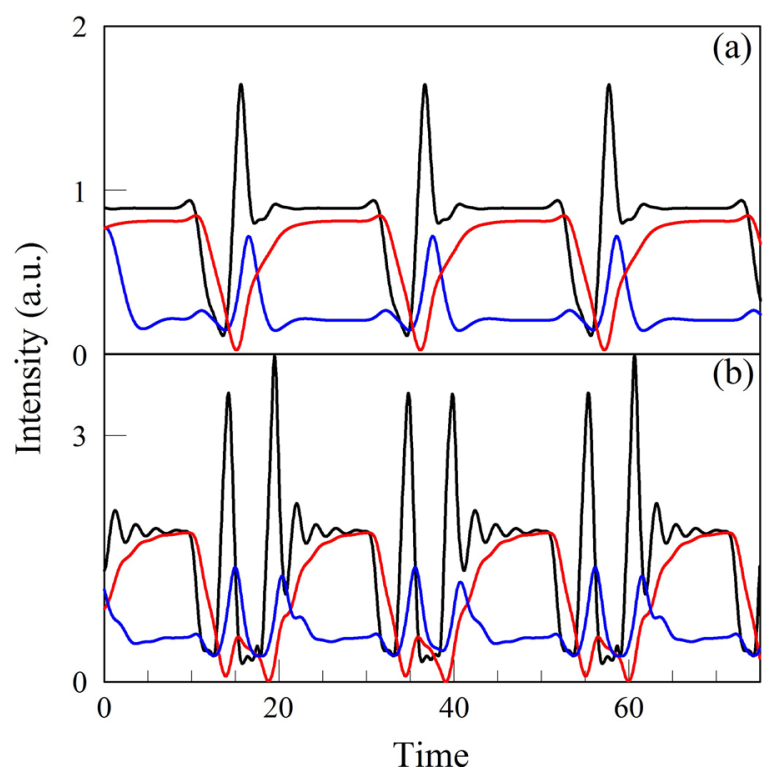

FIG. 11. Numerically obtained fundamental pulse train (a) and duplet trace (b). In both (a) and (b), the upper (black) curve shows the full intensity of the system. The lower (red) curve shows the filtered intensity for the filter located at the most powerful modes. The lowest (blue) curve shows the filtered intensity with the filter located on one wing of the spectrum centred at zero frequency. In each case, the filter had a bandwidth of 0.5 . 


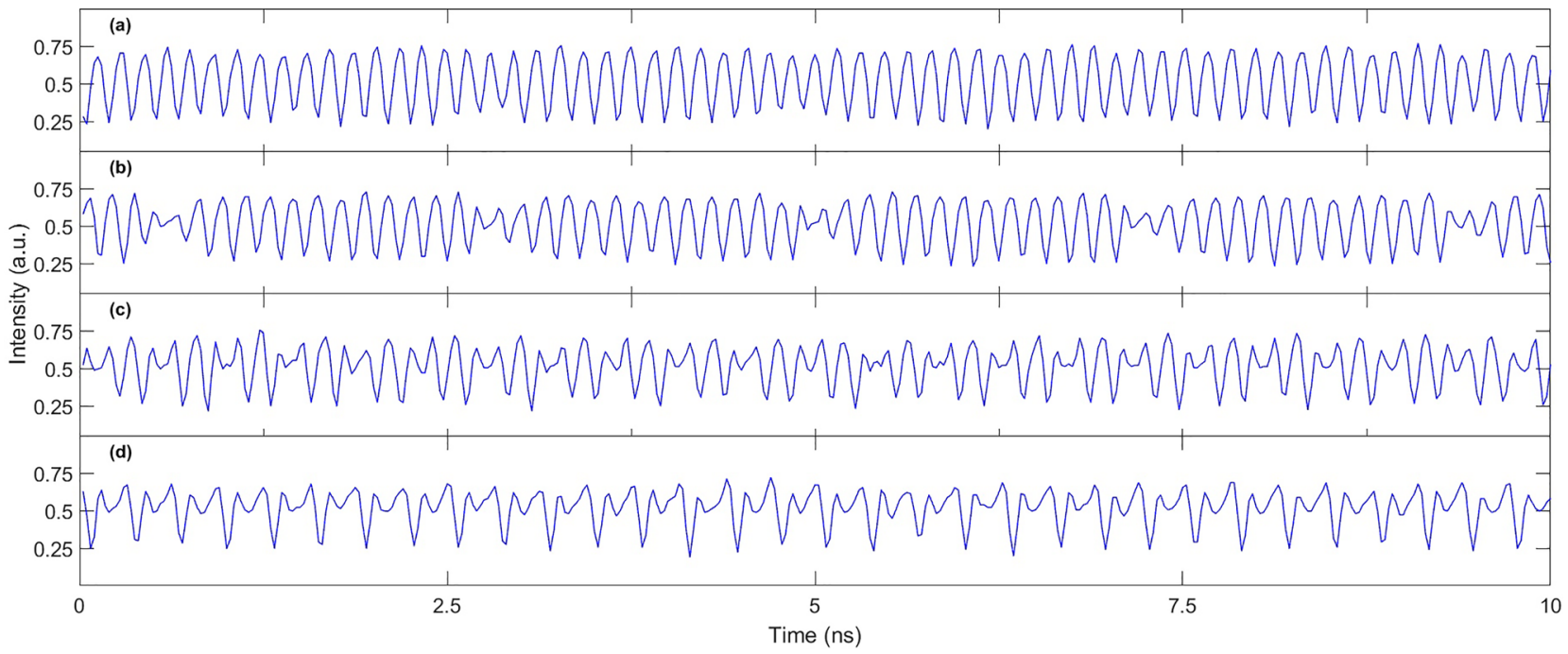

FIG. 12. Times series of the QW laser are shown for increasing feedback.

\section{QUANTUM WELL DEVICE}

In this section, we demonstrate the generality of the phenomenon by investigating the behaviour of a different device type. The device used is a multi-quantum well device with a threshold of $9 \mathrm{~mA}$ pumped at $25 \mathrm{~mA}$. Its relaxation oscillation frequency is found to be $7.6 \mathrm{GHz}$ at this current. Again, the output is analyzed using a fast real-time oscilloscope with a $12 \mathrm{GHz}$ photodetector. The setup is schematically represented by Fig. 1 just as for the QD case. The cavity used has a length of $\sim 34 \mathrm{~cm}$ which corresponds to an ECRR of $440 \mathrm{MHz}$. The feedback is controlled by rotating a quarterwave plate relative to a fixed linear polarizer which is aligned to the natural polarization of the laser.

The evolution of the output with increasing feedback strength depends strongly on the initial conditions ${ }^{12}$ and the ECM chosen, in particular. We show in Figs. 12 and 13 the sequence of behaviour observed in our experiment. In Fig. 12(a), a stable limit cycle appears with an oscillation close to the RO frequency just as in the QD case. In Fig. 12(b), a periodic trace is obtained with round trip period. Within each round trip is a packet of oscillations at the RO frequency. Each packet is separated by the absence of a single oscillation in the intensity. We believe this to be analogous to the trace in Fig. 2(b). The round trip period is found in each case and while there are marked differences in the behaviours within each round trip we attribute this to the different physics of the device type. In the QD case, the typical TEP shape is observed. In the QW case, prominent RO frequency oscillations are found, potentially indicative of the weaker damping of such devices or related to different phase relationships between the ECMs.

Increasing the feedback strength, the output is modified. Figure $12(d)$ is a period doubled version of (a). Figure 12(c) shows an alternating pattern of the period doubling and the regular limit cycle oscillations as found in (a).

Figure 13 shows the FFT for each time series shown in Fig. 12. The peak in the FFT is around the RO frequency of the laser in each case and the shortest separation between peaks is the ECRR frequency. The separation between the

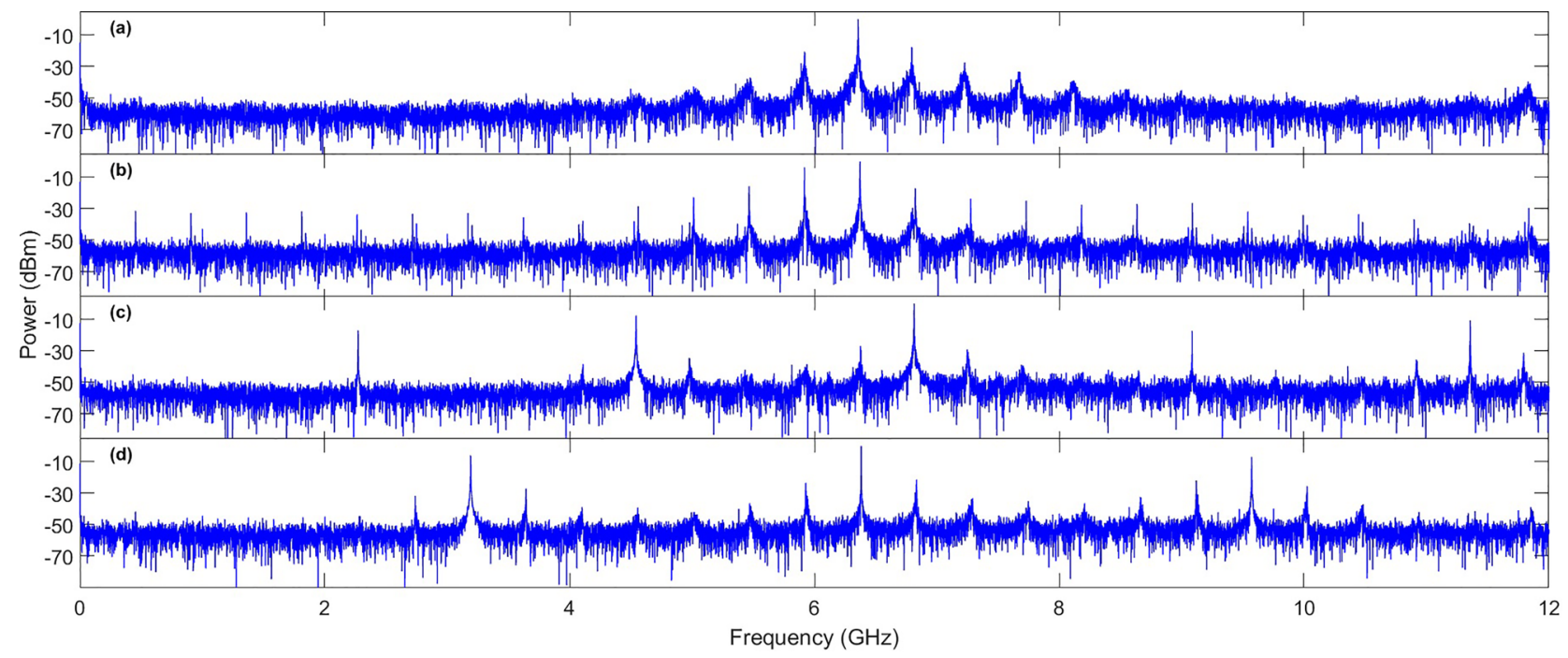

FIG. 13. FFTs of the time series from the QW laser. These subplots correspond to the subplot of the same letter in Fig. 12. 
main peaks in the FFT for (c) and (d) is given by $1 / 3$ and $1 / 2$ of the RO frequency, respectively, suggesting locking at submultiples of the RO frequency in the dynamics. Increasing the feedback still further yields a chaotic output.

\section{A. Relaxation oscillation damping}

In the case of the QD devices, the high damping of the ROs is known to endow the devices with an enhanced stability under feedback. ${ }^{13}$ It is this enhanced stability that allows the observation of the resonance phenomenon. With high damping, it takes a significant amount of feedback to undamp the ROs. This means that the feedback can be sufficiently high to allow the generation of ECMs before the RO undamping and the resonant features can thus emerge. For weakly damped devices, the ROs become undamped at low feedback levels, before the ECMs arise and this prevents the mutual locking of the frequencies. A small signal modulation analysis of our device allowed us to calculate the RO frequency and damping. As already stated, for the pump current in this work, the RO frequency is approximately $7.6 \mathrm{GHz}$ and we approximate the damping at $22 \mathrm{GHz}$. This is significantly higher than that of conventional devices and is what allows the requisite high feedback levels. This strongly suggests that our resonance locking phenomenon should be visible with other devices with similarly high damping levels.

\section{CONCLUSION}

As shown in Ref. 19, a spontaneous resonant modelocking can be obtained with single mode QD lasers undergoing optical feedback. We have analysed this effect in much greater detail here. The first Hopf bifurcation of the steady state is at a frequency close to the RO frequency but seems to occur at a nearby ECM frequency. Further increasing the feedback strength leads to increasingly complicated multipulse trains before finally yielding a chaotic output. This appears to arise via a splintering of modal groups (and consequently the trailing edge plateaux) similar to that shown in Ref. 22.

To demonstrate that the underlying physics of the locking is not unique to QD devices, we also analysed a QW device. This device also has a high damping compared with conventional semiconductor lasers. Similar dynamics were obtained with an initial instability close to the RO frequency followed by increasingly complicated pulse trains. In contrast to the QD results, trailing edge plateaux were not observed with this device confirming the identification of QD physics in their creation. In both cases, we confirmed that the experimental results are qualitatively unchanged for slight changes in the optical phase by making slight changes in the mirror position.

The most important feature for the devices seems to be the high RO damping. In addition to this, having a long external cavity is beneficial for the locking since the ECMs are relatively close and thus the RO frequency is always relatively close to an ECM. We suggest that the phenomenon is generic once these two criteria hold. An analysis of other highly damped (yet still Class B) lasers would be very interesting in this regard. We note that a phase-locking of ECMs was recently obtained in a theoretical model for QC lasers. ${ }^{18}$ The absence of ROs was cited as a major contributing factor in this work suggesting that it is a similar phenomenon to the results in this paper.

The novel, staircase-like evolution of the instability frequency suggests that the dependence of the RO frequency on the feedback level may warrant further analysis. ${ }^{25}$

${ }^{1}$ R. W. Tkach and A. R. Chraplyvy, IEEE J. Lightwave Technol. 4, 1655 (1986).

${ }^{2}$ T. Heil, I. Fischer, and W. Elsäßer, J. Opt. B2, 413 (2000).

${ }^{3}$ E. Sooudi, C. de Dios, J. G. McInerney, G. Huyet, F. Lelarge, K. Merghem, R. Rosales, A. Martinez, A. Ramdane, and S. P. Hegarty, IEEE J. Sel. Top. Quantum Electron. 19(4), 1101208 (2013).

${ }^{4}$ C. Otto, K. Lüdge, A. G. Vladimirov, M. Wolfrum, and E. Schöll, New J. Phys. 14, 113033 (2012).

${ }^{5}$ D. Lenstra, B. Verbeek, and A. J. Den Boef, IEEE J. Quantum Electron. QE-21, 674-679 (1985)

${ }^{6} \mathrm{~J}$. Mørk, B. Tromborg, and J. Mark, IEEE J. Quantum Electron. 28, 93-108 (1992).

${ }^{7}$ T. Sano, Phys. Rev. A 50, 2719 (1994).

${ }^{8}$ S. Azouigui, B. Kelleher, S. P. Hegarty, G. Huyet, B. Dagens, F. Lelarge, A. Accard, D. Make, O. Le Gouezigou, K. Merghem, A. Martinez, Q. Zou, and A. Ramdane, Opt. Express 15, 014155 (2007).

${ }^{9}$ J. Mørk, J. Mark, and B. Tromborg, Phys. Rev. Lett. 65, 1999-2002 (1990).

${ }^{10} \mathrm{H}$. Li, J. Ye, and J. G. McInerney, IEEE J. Quantum Electron. 29, 2421 (1993).

${ }^{11}$ K. Petermann, IEEE J. Sel. Top. Quantum Electron. 1, 480 (1995).

${ }^{12}$ A. Locquet, B. Kim, D. Choi, N. Li, and D. S. Citrin, Phys. Rev. A 95, 023801 (2017).

${ }^{13}$ D. O’Brien, S. P. Hegarty, G. Huyet, and A. V. Uskov, Opt. Lett. 29, 1072 (2004).

${ }^{14}$ K. Lüdge, M. J. P. Bormann, E. Malić, P. Hövel, M. Kuntz, D. Bimberg, A. Knorr, and E. Schöll, Phys. Rev. B 78, 035316 (2008).

${ }^{15}$ T. Heil, I. Fischer, and W. Elsäßer, Phys. Rev. A 58, R2672 (1998).

${ }^{16}$ T. Erneux, V. Kovanis, and A. Gavrielides, Phys. Rev. E 88, 032907 (2013).

${ }^{17}$ C. Wang, F. Grillot, V. Kovanis, and J. Even, J. Appl. Phys. 113, 063104 (2013).

${ }^{18}$ L. L. Columbo and M. Brambilla, Opt. Express 22, 10105 (2014).

${ }^{19}$ B. Tykalewicz, D. Goulding, S. P. Hegarty, G. Huyet, T. Erneux, B. Kelleher, and E. A. Viktorov, Opt. Express 24, 4239 (2016).

${ }^{20}$ Similar traces to Fig. 2 were shown in Ref. 19. We show them here to allow this expanded manuscript to be self-contained.

${ }^{21}$ M. Radziunas, A. G. Vladimirov, E. A. Viktorov, G. Fiol, H. Schmeckebier, and D. Bimberg, Appl. Phys. Lett. 98, 031104 (2011).

${ }^{22}$ E. A. Viktorov, T. Habruseva, S. P. Hegarty, G. Huyet, and B. Kelleher, Phys. Rev. Lett. 112, 224101 (2014).

${ }^{23}$ F. Kéfélian, S. ODonoghue, M. T. Todaro, J. G. McInerney, and G. Huyet, IEEE Photon. Technol. Lett. 20, 1405 (2008).

${ }^{24}$ A. G. Vladimirov and D. Turaev, Phys. Rev. A 72, 033808 (2005).

${ }^{25}$ J. Ohtsubo, Semiconductor Lasers: Stability, Instability and Chaos (Springer Series in Optical Sciences, 2013). 